\title{
Review Article \\ Oxidative Stress and Vascular Damage in Hypertension: Role of Angiotensin II
}

\author{
Agostino Virdis, Emiliano Duranti, and Stefano Taddei \\ Department of Internal Medicine, University of Pisa, 56100 Pisa, Italy \\ Correspondence should be addressed to Agostino Virdis, a.virdis@med.unipi.it
}

Received 18 February 2011; Accepted 16 March 2011

Academic Editor: Isabella Sudano

Copyright () 2011 Agostino Virdis et al. This is an open access article distributed under the Creative Commons Attribution License, which permits unrestricted use, distribution, and reproduction in any medium, provided the original work is properly cited.

\begin{abstract}
Reactive oxygen species are oxygen derivates and play an active role in vascular biology. These compounds are generated within the vascular wall, at the level of endothelial and vascular smooth muscle cells, as well as by adventitial fibroblasts. In healthy conditions, ROS are produced in a controlled manner at low concentrations and function as signaling molecules regulating vascular contraction-relaxation and cell growth. Physiologically, the rate of ROS generation is counterbalanced by the rate of elimination. In hypertension, an enhanced ROS generation occurs, which is not counterbalanced by the endogenous antioxidant mechanisms, leading to a state of oxidative stress. In the present paper, major angiotensin II-induced vascular ROS generation within the vasculature, and relative sources, will be discussed. Recent development of signalling pathways whereby angiotensin II-driven vascular ROS induce and accelerate functional and structural vascular injury will be also considered.
\end{abstract}

\section{Introduction}

Hypertension is associated with increased peripheral resistance, resulting predominantly from functional, structural, and mechanical alterations at the level of small-resistance arteries. Functional alterations, which include an impaired endothelial function, are mainly assessed as an impaired acetylcholine-induced, endothelium-dependent relaxation. Vascular structural changes include vascular remodeling, secondary to an increased cell growth, cell migration, and low-grade vascular inflammation $[1,2]$. In particular, an increased media-to-lumen ratio $(\mathrm{M} / \mathrm{L})$ may result from a reduced outer diameter that narrows the lumen without net growth (eutrophic remodeling) or from a thicker media encroaching on the lumen (hypertrophic remodeling) $[1,2]$.

Another hallmark of hypertension-induced structural abnormalities is represented by changes in the mechanical properties of arteries, with particular regard for increased stiffness [3]. Vascular fibrosis is critically important in the determinism of vascular structural modifications, and it involves changes in extracellular matrix (ECM) components, including collagen type I and III, elastin, and fibronectin. An increase in collagen and fibronectin and a decrease in elastin contents have been shown in the media of small arteries from hypertensive animals [3-5].

It is widely accepted that angiotensin (Ang) II, traditionally involved in modulating blood pressure and electrolyte homeostasis, is also greatly implicated in the pathogenesis of endothelial dysfunction and vascular remodeling [6-8]. This concept is strengthened by the evidence that chronic AT1 receptor blockade is able to correct the altered structure and endothelial dysfunction of subcutaneous resistance small vessels from patients with essential hypertension whereas the $\beta$-receptor blockade has no effect, despite similar blood pressure lowering effect [9]. Major mechanisms whereby Ang II exerts vascular damage include generation of reactive oxygen species (ROS) and stimulation of redox-dependent signalling pathways $[6,10,11]$.

The present review will focus on major Ang II-induced vascular ROS generation and on recent development of signalling pathways whereby Ang II-driven vascular ROS induce and accelerate functional and structural vascular injury. 


\section{Reactive Oxygen Species in Vascular Wall}

ROS are ubiquitous reactive derivatives of $\mathrm{O}_{2}$ metabolism found in the environment and in all biological systems. ROS are implicated in many intracellular signaling pathways leading to changes in gene transcription and protein synthesis and consequently in cell function. Within the cardiovascular system, ROS play a crucial physiological role in maintaining cardiac and vascular integrity and a pathophysiological role in cardiovascular dysfunction associated with several clinical conditions, including hypertension [12, 13]. The most important ROS detectable within the vasculature include the superoxide anion $\left({ }^{\circ} \mathrm{O}_{2}{ }^{-}\right)$, hydrogen peroxide $\left(\mathrm{H}_{2} \mathrm{O}_{2}\right)$, hydroxyl radical $\left({ }^{\bullet} \mathrm{OH}\right)$, and the reactive nitrogen species peroxynitrite $\left(\mathrm{ONOO}^{-}\right)$, which have been regarded as a nasty, life-threatening, and destructive oxygen-derived toxicant. In healthy conditions, ROS are produced in a controlled manner at low concentrations and function as signaling molecules regulating vascular contraction-relaxation and cell growth [14]. Physiologically, ROS generation is tightly regulated by endogenous cellular antioxidants, which include superoxide dismutase (SOD), catalase, thioredoxin, glutathione, and antioxidant vitamins. In physiological conditions, the rate of ROS generation is counterbalanced by the rate of elimination. In contrast, under pathological conditions, such as hypertension, ROS are produced in concentrations that cannot be controlled by the usual protective antioxidant mechanisms employed by the cells, leading to a state of oxidative stress [13]. Indeed, when produced in excess, $\bullet^{\cdot} \mathrm{O}_{2}{ }^{-}$reacts with nitric oxide $(\mathrm{NO})$ to produce a dramatic concentration of the toxic $\mathrm{ONOO}^{-}$which promotes a variety of negative effects on cellular function. These include alteration of transcription factors, kinases, protein synthesis, and redox-sensitive genes, which in turn influence endothelial function, increase vascular contractility, vascular smooth muscle cell growth and apoptosis, monocyte migration, lipid peroxidation, inflammation, and increased deposition of ECM proteins, all major processes deeply involved in the pathogenesis and progression of vascular damage in cardiovascular disease $[15,16]$.

\section{Major Sources of ROS Generation in the Vascular Wall}

Vascular ROS may be produced at the level of endothelial, as well as smooth muscle and adventitial cells, and can be generated by several enzymes. As concerns hypertensionrelated vascular disease, major sources of ROS are xanthine oxidase, uncoupled endothelial $\mathrm{NO}$ synthase, $\mathrm{NAD}(\mathrm{P}) \mathrm{H}$ oxidase, and cyclooxygenase (COX) [17]. The role of COX in the Ang II-mediated oxidant excess will be described later.

The xanthine oxidase system, detectable in the vascular endothelium, catalyses the oxidation of hypoxanthine and xanthine to form ${ }^{\cdot} \mathrm{O}_{2}{ }^{-}$. Major demonstrations of the role of xanthine oxidase-system concern the setting of ischemiareperfusion injury and heart failure. Nevertheless, some experimental reports also indicate an involvement of such complex in hypertension. In mesenteric small vessels from spontaneously hypertensive rats (SHR), an enhanced activity of xanthine oxidase was documented [18]. Beyond its effect within the vascular wall, an active role of xanthine oxidase has been also documented in the kidney from SHR or Dahl salt-sensitive rats. Usually, the xanthine oxidase enzymatic system is inhibited by allopurinol. Of note, chronic allopurinol administration in SHR, while abrogating the renal xanthine oxidase-related ROS generation, failed to reduce blood pressure values, suggesting that, at least in such animal model of disease, the increased renal ROS production is a mere consequence of hypertension rather than a pathogenetic factor [19]. This concept is supported by the findings that in these animals chronic allopurinol intake is also associated with an amelioration of cardiac hypertrophy [20].

Endothelial nitric oxide synthase (eNOS), the enzymatic system constitutively delegated to produce NO, can also generate ROS in those conditions characterized by deficiency of the substrate arginine or the cofactor tetrahydrobiopterin $\left(\mathrm{BH}_{4}\right)$. This process, which has been called "NOS uncoupling," implies that the physiological activity of the enzyme for NO production is decreased and switched to the NOSdependent ${ }^{\cdot} \mathrm{O}_{2}{ }^{-}$generation. eNOS uncoupling has been documented in several pathological conditions, including diabetes [21], hyperhomocysteinemia [22], and hypertension [23]. eNOS uncoupling has been demonstrated in DOCA salt-induced hypertensive mice. In this animal model, the critical step in this uncoupling seems to be oxidation of $\mathrm{BH}_{4}$ by $\mathrm{ONOO}^{-}$, reducing the bioavailability of this critical cofactor [23]. The activity of eNOS uncoupling as ROS source in hypertension is strengthened by human evidence in diabetic and hypertensive patients, where endothelial oxidant excess was dramatically reduced by administration of the precursor of $\mathrm{BH}_{4}[24,25]$.

A large body of evidence indicated that $\mathrm{NAD}(\mathrm{P}) \mathrm{H}$ oxidase represents the major source of ROS in the vascular wall. The activation of such enzymatic system, which utilises NADH/NADPH as electron donor to reduce molecular oxygen and generate ${ }^{\cdot} \mathrm{O}_{2}{ }^{-}$, requires the assembly of cytosolic (p47phox, p67phox) and membrane-bound (gp91phox/Nox1/Nox4 and p22phox) subunits to form a functional enzyme complex [17]. In the vasculature the $\mathrm{NAD}(\mathrm{P}) \mathrm{H}$ oxidase complex is at least partly preassembled, as a significant proportion of $\mathrm{NAD}(\mathrm{P}) \mathrm{H}$ oxidase subunits are colocalized intracellularly in endothelial cells [17]. It is widely recognized that $\mathrm{NAD}(\mathrm{P}) \mathrm{H}$ oxidase is the primary source of ${ }^{\bullet} \mathrm{O}_{2}{ }^{-}$in the vasculature [15], and it is functionally active either in the endothelium or in the media and adventitia as well [15]. Within the vascular wall, all the $\mathrm{NAD}(\mathrm{P}) \mathrm{H}$ oxidase subunits are expressed, to varying degrees $[15,17]$.

\section{Role of Angiotensin II on ROS Generation within the Vasculature}

A large body of evidence supports a crucial role for ROS production, particularly from $\mathrm{NAD}(\mathrm{P}) \mathrm{H}$ oxidase, in vascular injury which characterizes the hypertensive disease. Ang II represents one of the major vasoactive peptides, together 
with cytokines and growth factors, involved in the regulation and activation of $\mathrm{NAD}(\mathrm{P}) \mathrm{H}$ oxidase. Ang II stimulates activation of $\mathrm{NAD}(\mathrm{P}) \mathrm{H}$ oxidase, increases expression of $\mathrm{NAD}(\mathrm{P}) \mathrm{H}$ oxidase subunits, and induces ROS generation in vascular smooth muscle cells, endothelial cells, adventitial fibroblasts, and intact arteries as well $[15,26]$. Experimental reports documented that Ang II may exert such effects via AT1 receptors. Of note, ROS may regulate AT1 receptor gene expression, which in turn modulates ROS generation, thus perpetuating a vicious circle [27]. Molecular mechanisms and signaling pathways whereby Ang II-derived NAD $(\mathrm{P}) \mathrm{H}$ oxidase activation leads to vascular cell oxidation are beyond the scope of this report and, therefore, the reader is directed to many excellent reviews focusing on these aspects $[6,17]$.

The strict crosstalk between Ang II and $\mathrm{NAD}(\mathrm{P}) \mathrm{H}$ oxidase is confirmed by in vivo studies. Thus, in Ang II-infused hypertensive rats, $\mathrm{NAD}(\mathrm{P}) \mathrm{H}$ oxidase subunit expression and activity are increased, and administration of an $\mathrm{NAD}(\mathrm{P}) \mathrm{H}$ oxidase inhibitor reduces vascular ${ }^{\bullet} \mathrm{O}_{2}{ }^{-}$generation $[28,29]$. More recently, it was demonstrated that chronic administration of apocynin, a selective inhibitor of $\mathrm{NAD}(\mathrm{P}) \mathrm{H}$ oxidase, prevents the increase in media-to-lumen ratio, an index of vascular remodeling, as well as endothelial dysfunction and collagen deposition at the level of mesenteric resistance arteries from Ang II-infused mice [30]. These data suggest that activation of vascular $\mathrm{NAD}(\mathrm{P}) \mathrm{H}$ oxidase plays an important role in vascular functional and structural changes that accompany development of hypertension in Ang II-infused mice. Of note, in this study, apocynin, which, abolishing the Ang II-induced increase of vascular $\mathrm{NAD}(\mathrm{P}) \mathrm{H}$ oxidase activity, was able to attenuate the systolic blood pressure rise induced by Ang II [30]. These findings demonstrate that $\mathrm{NAD}(\mathrm{P}) \mathrm{H}$ oxidase inhibition leads to blood pressure reduction in an Ang II-dependent hypertensive murine model and support the concept that $\mathrm{NAD}(\mathrm{P}) \mathrm{H}$ oxidasederived ${ }^{\bullet} \mathrm{O}_{2}{ }^{-}$plays a role in the Ang II-induced blood pressure elevation. It is worth noting that in this experimental condition, apocynin, while only partially reducing blood pressure, totally abolished the Ang II-mediated NAD $(\mathrm{P}) \mathrm{H}$ oxidase increased activity, thus suggesting that, at least in mice, redox-independent pathways also underlie Ang IIinduced blood pressure elevation. These findings, obtained by pharmacological doses of exogenous Ang II, are supported by other reports assessing the role for the endogenous reninangiotensin system in increasing ROS production during hypertension. In the 2-kidney 1-clip model of renovascular hypertension (a model characterized by a high activation of the renin-angiotensin system), endothelial dysfunction was associated with an $\mathrm{NAD}(\mathrm{P}) \mathrm{H}$ oxidase-derived increased - $\mathrm{O}_{2}{ }^{-}$production, a condition which in part participates in blood pressure elevation in blood pressure [31]. In saltsensitive Dahl rats, another animal model of hypertension characterized by activation of the local renin-angiotensin system, chronic administration with an Ang II receptor blocker dramatically reduced vascular ${ }^{\circ} \mathrm{O}_{2}{ }^{-}$production [32]. Of note, in animal model of salt-sensitive hypertension, treatment with a gp91phox-containing $\mathrm{NAD}(\mathrm{P}) \mathrm{H}$ oxidase inhibitor also prevented the increased vascular ${ }^{\cdot} \mathrm{O}_{2}{ }^{-}$production together with the expression of proinflammatory molecules, thus supporting the concept of a linkage between renin-angiotensin system, $\mathrm{NAD}(\mathrm{P}) \mathrm{H}$ oxidase activity, and vascular inflammation [33]. Importantly, the deleterious effect by Ang II-derived NAD(P)H oxidase activity on vasculature is not dependent on blood pressure elevation. Indeed, it was documented that in an animal model of Ang II-hypertension, production of $\mathrm{H}_{2} \mathrm{O}_{2}$, while essential for Ang II-mediated vascular changes, had no significant impact on blood pressure [34].

There is also evidence for ROS involvement in the hypertension-related vascular disease independent of Ang II actions. Indeed, it has been suggested that endothelin-1 (ET-1) is implicated in the development of vascular changes through the ROS generation via $\mathrm{NAD}(\mathrm{P}) \mathrm{H}$ oxidase activation [35]. Interestingly, the ET-1-mediated enhanced ROS production can affect vascular structure and function independently of blood pressure modifications. Direct infusion of ET-1 can increase $\mathrm{NAD}(\mathrm{P}) \mathrm{H}$ oxidase-dependent ${ }^{\cdot} \mathrm{O}_{2}{ }^{-}$ production, which is not significantly related to the development of hypertension [36]. Recently, an animal model of transgenic mice overexpressing human preproET-1 gene specifically in blood vessel endothelium was generated. This is an useful model to investigate the role of endotheliumgenerated ET-1 on the endothelial function and vascular structure of small resistance arteries. These animals exhibited 3-fold higher vascular tissue ET-1 mRNA and 7-fold higher ET-1 plasma levels than did wild-type mice but no significant elevation in blood pressure [37]. Despite the absence of significant blood pressure elevation, transgenic mice exhibited marked hypertrophic remodeling and oxidant excessdependent endothelial dysfunction of resistance vessels, altered ET-1 and ET-3 vascular responses, and significant increases in $\mathrm{ET}_{B}$ expression compared with wild-type littermates. Moreover, transgenic mice generated significantly higher oxidative stress, possibly through increased activity and expression of vascular $\mathrm{NAD}(\mathrm{P}) \mathrm{H}$ oxidase [37]. Using a transgenic approach, these findings represent the demonstration that endothelium-secreted human ET-1 induces vascular remodeling and endothelial dysfunction in the absence of significant increases in blood pressure, as also evidenced above for Ang II actions.

In conclusions, these data unambiguously underscore the concept that Ang II exerts several pleiotropic deleterious vascular effects, including functional and structural changes, through $\mathrm{NAD}(\mathrm{P}) \mathrm{H}$ oxidase-derived ROS generation, an effect which does not necessarily occur as a consequence of blood pressure elevation.

\section{Role of Cyclooxygenase on Ang II-Induced Vascular Damage}

In the last decade, a growing literature provided evidence that a major pathway involved in vascular disease is cyclooxygenase (COX) activity [38]. Indeed, COX metabolizes arachidonic acid from membrane-bound phospholipids into the unstable intermediate $\mathrm{PGH}_{2}$, which, in turn, is converted by an array of downstream enzymes to form a variety of bioactive prostaglandins (PGs) and thromboxane (TX) $\mathrm{A}_{2}$, 
collectively termed prostanoids [39]. These have been considered autacoids mediating a variety of responses in the cardiovascular system, including modulation of vascular tone and structure and inflammatory responses. Under physiological conditions, prostacyclin $\left(\mathrm{PGI}_{2}\right)$ is the major prostanoid identified and released by endothelial cells mediating several protective effects on the vascular wall, including relaxation and inhibition of platelet aggregation and adhesion [40]. The predominant opponent of $\mathrm{PGI}_{2}$ is $\mathrm{TXA}_{2}$, specifically acting on thromboxane-prostanoid (TP) receptors mainly located on smooth muscle cells where it causes vasoconstriction [41]. Under pathological conditions, such as inflammation or atherosclerosis, the $\mathrm{PGI}_{2}$ production decreases, and COXderived vasoconstrictor substance release, including $\mathrm{TXA}_{2}$, becomes predominant. Two distinct COX isoenzymes, COX1 and COX-2, have been described. Although COX-1 is constitutively expressed to produce physiologically relevant prostanoids, COX-2 is regarded as an inducible isoform, which can be rapidly upregulated by a number of stimuli, A direct interaction between Ang II and the COX pathway has been described. Thus, a direct interaction between Ang II and COX-2 pathway was documented in in vitro studies, either in vascular smooth muscles (VSMCs) or endothelial cells. Ohnaka et al. [42] observed that Ang II dose-dependently increased the expression of COX-2 mRNA in cultured rat VSMCs. This effect was totally abolished by the $\mathrm{AT}_{1}$ receptor antagonist losartan, by the mitogen-activated protein kinase (MAPK) kinase-1 inhibitor, and by the p38 MAPK inhibitor. A similar possibility was hypothesized by Young et al. [43], who documented that a COX-2-derived prostanoid, possibly $\mathrm{TXA}_{2}$, may contribute to VSMC hyperplasia in rat aortic injury or pathophysiological conditions associated with elevated levels of TNF- $\alpha$ or Ang II. In this study, the activation of MAP kinase was confirmed to be implicated as a signaling pathway for COX-2 gene transcription and proliferative response of VSMCs to Ang II. An increased COX2 expression secondary to Ang II incubation was confirmed also in cultured human VSMCs [44]. Taken together, these in vitro animal and human reports provide evidence that Ang II-mediated activation of $\mathrm{AT}_{1}$ receptors plays a crucial role in triggering multiple biological responses in VSMCs, including cell growth and proliferation, via increased COX2 expression. The relationship between Ang II and COX2 were extended in vivo. In a rat model of Ang II-induced hypertension, it was demonstrated that Ang II-mediated vascular lesions were associated with increased expression of COX-2 in the media of coronary arteries [45]. However, these findings were not confirmed when the interaction between Ang II and COX-2 were investigated in mREN2 rats, a transgenic animal model of hypertension characterized by Ang II-induced vascular and tissue injury [46]. In such animals, the authors explored whether COX-derived prostanoids were involved in the pathogenesis of Ang II-induced vascular injury, in myocardium and kidney. The main finding of this report was that neither nonselective COX inhibitor nor the COX-2 selective blocker was able to prevent Ang II-induced vascular and tissue damage. Moreover, myocardial and renal COX-2 mRNA expressions were decreased in mREN2 rats. Therefore, these results seem to exclude, at least in this particular transgenic animal model, a central role by COX in the pathogenesis of ANG II-induced vascular or end-organ damage. Of note, the interpretation of these findings deserve caution if we consider that this particular transgenic animal model does not reproduce physiological conditions. Nevertheless, when Ang II and COX interaction is investigated in resistance arteries, in more physiological conditions, a pivotal role of COX-1 on Ang II-mediated vascular changes, instead of COX-2, emerged. Thus, we documented that in mesenteric small vessels from Ang II-infused mice, COX-1 inhibition as well as TP receptor antagonist similarly improved the blunted endothelium-dependent relaxation to acetylcholine while COX-2 inhibition was ineffective [8]. In addition, a COX-2 downregulation and a simultaneous induction of COX-1 expression and staining in Ang II-vessels were detected. Concomitantly, the NAD $(\mathrm{P}) \mathrm{H}$ oxidase apocynin, while normalizing endothelial function, failed to modify the COX-2 downregulation and COX-1 upregulation [8]. Overall, these results demonstrate the participation of COX1 isoenzyme in the development of functional alterations of resistance arteries from Ang II-infused mice. As summarized in Figure 1, it was proposed that Ang II is associated to COX1 overexpression and COX-2 downregulation while Ang IImediated ROS production stimulates COX-1 activity, but not overexpression, to produce a contracting prostanoid, acting as an agonist on TP receptors [8]. Of note, in this report COX-1 blockade improved only in part the Ang II-induced endothelial dysfunction, thus confirming the presence of a residual direct and specific COX-1-independent effect of Ang II on the vascular wall [8].

Ang II plays also a crucial role in inducing mechanical changes of arteries, with particular regard for increased stiffness, and in determining changes in ECM components within the vascular wall $[3-5,47]$. Indeed, an increased collagen and fibronectin depositions, together with a decreased elastin content, have been described in the media of small arteries from Ang II-infused animals [4, 5, 47]. In this context, experimental observations strongly suggest a predominant role of COX-1-derived activators of TP receptors in the development/progression of vascular atherosclerosis [48]. Therefore, a direct interaction between Ang II and COX1 pathway can be hypothesized as one of the mechanisms whereby Ang II induces vascular structural changes. In a very recent report, this possibility has been investigated in our laboratory, by utilizing two different experimental methodologies, such as the employment of selective COX isoforms inhibitors, chronically administered to Ang II-treated mice, and the utilization of homozygous mice carrying a targeted disruption of COX-1 gene. In murine mesenteric small vessels, we documented that Ang II-induced hypertrophic remodeling and arterial stiffness were partly reduced by chronic administration of COX-1 selective inhibitor SC-560, or by the TP receptor blockade, while not being affected by the COX-2 inhibitor DFU, thus indicating that COX-1, but not COX-2, contributes to the pathogenesis of vascular structural and mechanical changes elicited by Ang II [49]. These findings, obtained by a pharmacological approach, were strongly substantiated by results from COX-1-KO mice, in which Ang II failed to elicit vascular remodeling or 


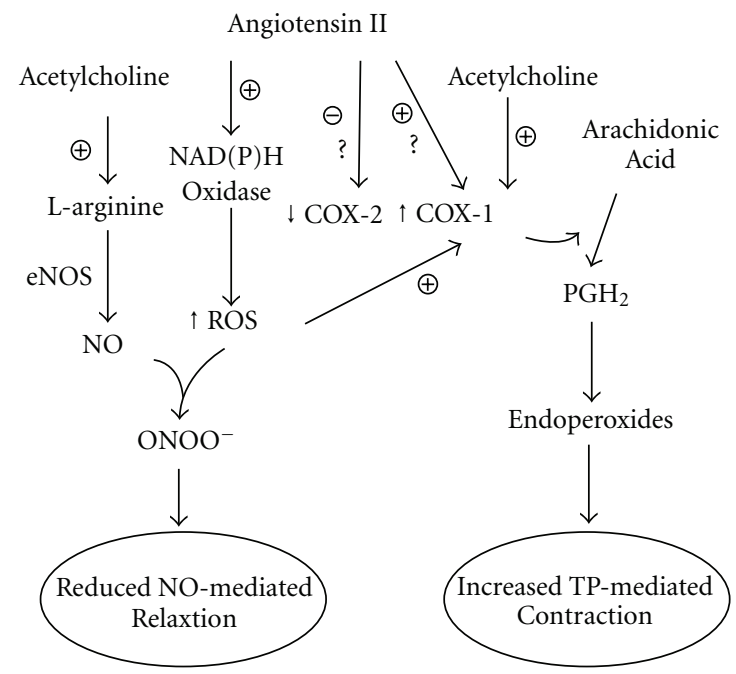

FIGURE 1: Hypothetic involvement of cyclooxygenase (COX) isoforms in angiotensin II- (AngII-) induced endothelial dysfunction. Ang II promotes reactive oxygen species (ROS) generation by vascular $\mathrm{NAD}(\mathrm{P}) \mathrm{H}$ oxidase activation. ROS rapidly react with nitric oxide (NO), leading to reduce its availability and to produce peroxynitrites $\left(\mathrm{ONOO}^{-}\right)$. Ang II might also directly downregulate COX-2 or upregulate COX-1. COX-1 is also activated by acetylcholine. Under such conditions, ROS stimulate COX-1 to transform arachidonic acid into endoperoxides, which in turn activate the contracting thromboxane-prostanoid (TP) receptors.

mechanical changes. In the same context, a significant role of the COX-1 pathway in Ang II-induced vascular ECM changes emerged. Indeed, the enhanced deposition of collagen and fibronectin was reduced by SC-560 and, to a similar extent, by the TP receptor antagonist, while not affected by DFU. In parallel, the vascular content of elastin was completely restored by COX-1 and TP receptor blockade, while not being ameliorated by COX-2 inhibition. In vessels from $C O X-1^{-/-}$mice, collagen, fibronectin, and elastin contents did not differ from wild type, and they were not modified by Ang II [49]. Overall, these findings unequivocally support a relevant role of COX-1-dependent TP receptor activation in the development of Ang II-induced hypertrophic vascular remodeling, changes in mechanics and ECM components, leading to vascular fibrosis.

In conclusions, although a strict interaction between Ang II and the COX pathway in the pathogenesis of vascular injury is well established, the pivotal role of COX-1 or COX2 isoforms may differ and predominate according to the experimental settings and the vascular districts considered. There is also the possibility that in several vascular beds a COX-1/COX-2 compensatory phenomenon occurs as a consequence of Ang II stimulation, leading to different patterns of prostanoid formation. Of course, the heterogeneous experimental models, including both the variety of cells stimulated and the different conditions in which Ang II is applied, contribute to complicate the picture. For these reasons, further experimental and clinical studies examining the interaction between the renin-angiotensin system and COX pathway are warranted.

\section{Conclusions}

ROS, including superoxide, hydrogen peroxide, and hydroxyl anion, and reactive nitrogen species, are the most important
$\mathrm{O}_{2}$ derivates, which play an active role in vascular biology. ROS are generated within the vascular wall, at the level of endothelial and vascular smooth muscle cells, as well as by adventitial fibroblasts. In healthy conditions, ROS are produced in a controlled manner at low concentrations and function as signaling molecules regulating vascular contraction-relaxation and cell growth. Physiologically, the rate of ROS generation is counterbalanced by the rate of elimination. Under pathological conditions, the ROS generation excess cannot be controlled by the usual protective antioxidant mechanisms, leading to a state of oxidative stress. A large body of evidence supports a crucial role for ROS production, particularly from NAD $(\mathrm{P}) \mathrm{H}$ oxidase, in vascular injury which characterizes the hypertensive disease. Ang II represents one of the major vasoactive peptides involved in the regulation and activation of $\mathrm{NAD}(\mathrm{P}) \mathrm{H}$ oxidase. Ang II stimulates activation of $\mathrm{NAD}(\mathrm{P}) \mathrm{H}$ oxidase, increases expression of $\mathrm{NAD}(\mathrm{P}) \mathrm{H}$ oxidase subunits, and induces ROS generation in vascular smooth muscle cells, endothelial cells, and adventitial fibroblasts. A large body of evidence from experimental and clinical studies unequivocally demonstrated that Ang II exerts several pleiotropic deleterious vascular effects, including functional and structural changes, through $\mathrm{NAD}(\mathrm{P}) \mathrm{H}$ oxidase-derived ROS generation. More recently, it was proposed that at the level of peripheral resistance arteries, Ang II-mediated ROS production stimulates COX1 activity, to produce contracting prostanoids(s), acting as agonists on TP receptors. Such mechanism likely contributes to the endothelial dysfunction and vascular atherosclerotic damage elicited by Ang II.

\section{References}

[1] E. L. Schiffrin, "Reactivity of small blood vessels in hypertension: relation with structural changes: state of the art lecture," Hypertension, vol. 19, no. 2, pp. II1-II9, 1992. 
[2] M. J. Mulvany, G. L. Baumbach, C. Aalkjaer et al., "Vascular remodeling," Hypertension, vol. 28, pp. 505-506, 1996.

[3] H. D. Intengan and E. L. Schiffrin, "Structure and mechanical properties of resistance arteries in hypertension: role of adhesion molecules and extracellular matrix determinants," Hypertension, vol. 36, no. 3, pp. 312-318, 2000.

[4] M. F. Neves, A. Virdis, and E. L. Schiffrin, "Resistance artery mechanics and composition in angiotensin II-infused rats: effects of aldosterone antagonism," Journal of Hypertension, vol. 21, no. 1, pp. 189-198, 2003.

[5] M. F. Neves, D. Endemann, F. Amiri et al., "Small artery mechanics in hyperhomocysteinemic mice: effects of angiotensin II," Journal of Hypertension, vol. 22, no. 5, pp. 959-966, 2004.

[6] R. M. Touyz and E. L. Schiffrin, "Signal transduction mechanisms mediating the physiological and pathophysiological actions of angiotensin II in vascular smooth muscle cells," Pharmacological Reviews, vol. 52, no. 4, pp. 639-672, 2000.

[7] A. Virdis, M. F. Neves, F. Amiri, E. Viel, R. M. Touyz, and E. L. Schiffrin, "Spironolactone improves angiotensin-induced vascular changes and oxidative stress," Hypertension, vol. 40, no. 4, pp. 504-510, 2002.

[8] A. Virdis, R. Colucci, M. Fornai et al., "Cyclooxygenase-1 is involved in endothelial dysfunction of mesenteric small arteries from angiotensin II-infused mice," Hypertension, vol. 49, no. 3, pp. 679-686, 2007.

[9] E. L. Schiffrin, J. B. Park, H. D. Intengan, and R. M. Touyz, "Correction of arterial structure and endothelial dysfunction in human essential hypertension by the angiotensin receptor antagonist losartan," Circulation, vol. 101, no. 14, pp. 1653 $1659,2000$.

[10] S. Rajagopalan, S. Kurz, T. Münzel et al., "Angiotensin IImediated hypertension in the rat increases vascular superoxide production via membrane NADH/NADPH oxidase activation. Contribution to alterations of vasomotor tone," Journal of Clinical Investigation, vol. 97, no. 8, pp. 1916-1923, 1996.

[11] A. Warnholtz, G. Nickenig, E. Schulz et al., "Increased NADHoxidase-mediated superoxide production in the early stages of atherosclerosis: evidence for involvement of the reninangiotensin system," Circulation, vol. 99, no. 15, pp. 20272033, 1999.

[12] K. K. Griendling, D. Sorescu, B. Lassègue, and M. UshioFukai, "Modulation of protein kinase activity and gene expression by reactive oxygen species and their role in vascular physiology and pathophysiology," Arteriosclerosis, Thrombosis, and Vascular Biology, vol. 20, no. 10, pp. 2175-2183, 2000.

[13] U. Landmesser and D. G. Harrison, "Oxidative stress and vascular damage in hypertension," Coronary Artery Disease, vol. 12, no. 6, pp. 455-461, 2001.

[14] R. M. Touyz and E. L. Schiffrin, "Ang II-stimulated superoxide production is mediated via phospholipase D in human vascular smooth muscle cells," Hypertension, vol. 34, no. 4, pp. 976-982, 1999.

[15] R. M. Touyz, F. Tabet, and E. L. Schiffrin, "Redox-dependent signalling by angiotensin II and vascular remodelling in hypertension," Clinical and Experimental Pharmacology and Physiology, vol. 30, no. 11, pp. 860-866, 2003.

[16] R. M. Touyz and E. L. Schiffrin, "Reactive oxygen species in vascular biology: implications in hypertension," Histochemistry and Cell Biology, vol. 122, no. 4, pp. 339-352, 2004.

[17] T. M. Paravicini and R. M. Touyz, "Redox signaling in hypertension," Cardiovascular Research, vol. 71, no. 2, pp. 247-258, 2006.
[18] H. Suzuki, F. A. Delano, D. A. Parks et al., "Xanthine oxidase activity associated with arterial blood pressure in spontaneously hypertensive rats," Proceedings of the National Academy of Sciences of the United States of America, vol. 95, no. 8, pp. 4754-4759, 1998.

[19] J. Laakso, E. Mervaala, J. J. Himberg et al., "Increased kidney xanthine oxidoreductase activity in salt-induced experimental hypertension," Hypertension, vol. 32, no. 5, pp. 902-906, 1998.

[20] J. T. Laakso, T. L. Teräväinen, E. Martelin, T. Vaskonen, and R. Lapatto, "Renal xanthine oxidoreductase activity during development of hypertension in spontaneously hypertensive rats," Journal of Hypertension, vol. 22, no. 7, pp. 1333-1340, 2004.

[21] Z. Bagi and A. Koller, "Lack of nitric oxide mediation of flowdependent arteriolar dilation in type I diabetes is restored by sepiapterin," Journal of Vascular Research, vol. 40, no. 1, pp. 47-57, 2003.

[22] A. Virdis, M. Iglarz, M. F. Neves et al., "Effect of hyperhomocystinemia and hypertension on endothelial function in methylenetetrahydrofolate reductase-deficient mice," Arteriosclerosis, Thrombosis, and Vascular Biology, vol. 23, no. 8, pp. 1352-1357, 2003.

[23] U. Landmesser, S. Dikalov, S. R. Price et al., "Oxidation of tetrahydrobiopterin leads to uncoupling of endothelial cell nitric oxide synthase in hypertension," Journal of Clinical Investigation, vol. 111, no. 8, pp. 1201-1209, 2003.

[24] T. J. Guzik, S. Mussa, D. Gastaldi et al., "Mechanisms of increased vascular superoxide production in human diabetes mellitus: role of $\mathrm{NAD}(\mathrm{P}) \mathrm{H}$ oxidase and endothelial nitric oxide synthase," Circulation, vol. 105, no. 14, pp. 1656-1662, 2002.

[25] Y. Higashi, S. Sasaki, K. Nakagawa et al., “Tetrahydrobiopterin enhances forearm vascular response to acetylcholine in both normotensive and hypertensive individuals," American Journal of Hypertension, vol. 15, no. 4, pp. 326-332, 2002.

[26] K. K. Griendling, C. A. Minieri, J. D. Ollerenshaw, and R. W. Alexander, "Angiotensin II stimulates NADH and NADPH oxidase activity in cultured vascular smooth muscle cells," Circulation Research, vol. 74, no. 6, pp. 1141-1148, 1994.

[27] G. Nickenig, K. Strehlow, A. T. Bäumer et al., "Negative feedback regulation of reactive oxygen species on AT1 receptor gene expression," British Journal of Pharmacology, vol. 131, no. 4, pp. 795-803, 2000.

[28] T. Fukui, N. Ishizaka, S. Rajagopalan et al., "p22phox mRNA expression and NADPH oxidase activity are increased in aortas from hypertensive rats," Circulation Research, vol. 80, no. 1, pp. 45-51, 1997.

[29] F. E. Rey, M. E. Cifuentes, A. Kiarash, M. T. Quinn, and P. J. Pagano, "Novel competitive inhibitor of $\mathrm{NAD}(\mathrm{P}) \mathrm{H}$ oxidase assembly attenuates vascular $\mathrm{O}(2)(-)$ and systolic blood pressure in mice," Circulation Research, vol. 89, no. 5, pp. 408-414, 2001.

[30] A. Virdis, M. F. Neves, F. Amiri, R. M. Touyz, and E. L. Schiffrin, "Role of $\mathrm{NAD}(\mathrm{P}) \mathrm{H}$ oxidase on vascular alterations in angiotensin II-infused mice," Journal of Hypertension, vol. 22, no. 3, pp. 535-542, 2004.

[31] O. Jung, J. G. Schreiber, H. Geiger, T. Pedrazzini, R. Busse, and R. P. Brandes, "gp91phox-containing NADPH oxidase mediates endothelial dysfunction in renovascular hypertension," Circulation, vol. 109, no. 14, pp. 1795-1801, 2004.

[32] M. S. Zhou, A. G. Adam, E. A. Jaimes, and L. Raij, "In salt-sensitive hypertension, increased superoxide production is linked to functional upregulation of angiotensin II," Hypertension, vol. 42, no. 5, pp. 945-951, 2003. 
[33] M. S. Zhou, I. H. Schulman, P. J. Pagano, E. A. Jaimes, and L. Raij, "Reduced $\mathrm{NAD}(\mathrm{P}) \mathrm{H}$ oxidase in low renin hypertension: link among angiotensin II, atherogenesis, and blood pressure," Hypertension, vol. 47, no. 1, pp. 81-86, 2006.

[34] Y. Zhang, K. K. Griendling, A. Dikalova, G. K. Owens, and W. R. Taylor, "Vascular hypertrophy in angiotensin II-induced hypertension is mediated by vascular smooth muscle cellderived $\mathrm{H}_{2} \mathrm{O}_{2}$," Hypertension, vol. 46, no. 4, pp. 732-737, 2005.

[35] L. Li, G. D. Fink, S. W. Watts et al., "Endothelin-1 increases vascular superoxide via endothelin(A)-NADPH oxidase pathway in low-renin hypertension," Circulation, vol. 107, no. 7, pp. 1053-1058, 2003.

[36] A. A. Elmarakby, E. D. Loomis, J. S. Pollock, and D. M. Pollock, "NADPH oxidase inhibition attenuates oxidative stress but not hypertension produced by chronic ET-1," Hypertension, vol. 45, no. 2, pp. 283-287, 2005.

[37] F. Amiri, A. Virdis, M. F. Neves et al., "Endothelium-restricted overexpression of human endothelin-1 causes vascular remodeling and endothelial dysfunction," Circulation, vol. 110, no. 15, pp. 2233-2240, 2004.

[38] T. D. Warner and J. A. Mitchell, "Cyclooxygenases: new forms, new inhibitors, and lessons from the clinic," The FASEB Journal, vol. 18, no. 7, pp. 790-804, 2004.

[39] F. Cipollone, B. Rocca, and C. Patrono, "Cyclooxygenase-2 expression and inhibition in atherothrombosis," Arteriosclerosis, Thrombosis, and Vascular Biology, vol. 24, no. 2, pp. 246255,2004

[40] C. D. Funk, "Prostaglandins and leukotrienes: advances in eicosanoid biology," Science, vol. 294, no. 5548, pp. 1871-1875, 2001.

[41] D. Yang, M. Félétou, N. Levens, J. N. Zhang, and P. M. Vanhoutte, "A diffusible substance(s) mediates endotheliumdependent contractions in the aorta of SHR," Hypertension, vol. 41, no. 1, pp. 143-148, 2003.

[42] K. Ohnaka, K. Numaguchi, T. Yamakawa, and T. Inagami, "Induction of cyclooxygenase-2 by angiotensin II in cultured rat vascular smooth muscle cells," Hypertension, vol. 35 , no. 1 , pp. $68-75,2000$.

[43] W. Young, K. Mahboubi, A. Haider, I. Li, and N. R. Ferreri, "Cyclooxygenase-2 is required for tumor necrosis factor- $\alpha$ and angiotensin II-mediated proliferation of vascular smooth muscle cells," Circulation Research, vol. 86, no. 8, pp. 906-914, 2000.

[44] Z. W. Hu, R. Kerb, X. Y. Shi, T. Wei-Lavery, and B. B. Hoffman, "Angiotensin II increases expression of cyclooxygenase-2: implications for the function of vascular smooth muscle cells," Journal of Pharmacology and Experimental Therapeutics, vol. 303, no. 2, pp. 563-573, 2002.

[45] R. Rocha, C. L. Martin-Berger, P. Yang, R. Scherrer, J. Delyani, and E. McMahon, "Selective aldosterone blockade prevents angiotensin II/salt-induced vascular inflammation in the rat heart," Endocrinology, vol. 143, no. 12, pp. 4828-4836, 2002.

[46] H. Bohm, M. Lee, R. Kreutz et al., "Angiotensin II receptor blockade in TGR(mREN2)27: effects of renin-angiotensinsystem gene expression and cardiovascular functions," Journal of Hypertension, vol. 13, no. 8, pp. 891-899, 1995.

[47] P. Brassard, F. Amiri, and E. L. Schiffrin, "Combined angiotensin II type 1 and type 2 receptor blockade on vascular remodeling and matrix metalloproteinases in resistance arteries," Hypertension, vol. 46, no. 3, pp. 598-606, 2005.

[48] A. J. Cayatte, Y. Du, J. Oliver-Krasinski, G. Lavielle, T. J. Verbeuren, and R. A. Cohen, "The thromboxane receptor antagonist S18886 but not aspirin inhibits atherogenesis in apo E-deficient mice: evidence that eicosanoids other than thromboxane contribute to atherosclerosis," Arteriosclerosis, Thrombosis, and Vascular Biology, vol. 20, no. 7, pp. 17241728, 2000.

[49] A. Virdis et al., "63rd Annual High Blood Pressure Research Conference," Hypertension, vol. 54, p. e117, 2009, abstract no. P435. 


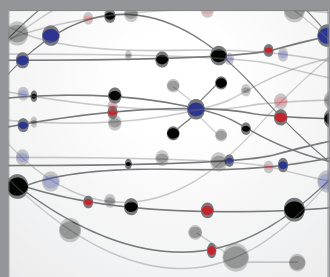

The Scientific World Journal
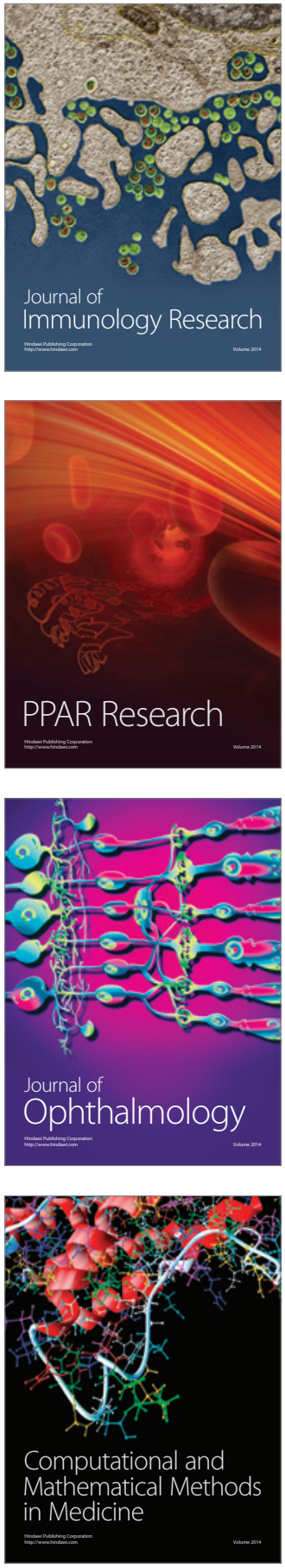

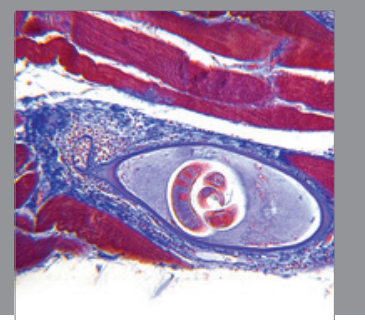

Gastroenterology

Research and Practice
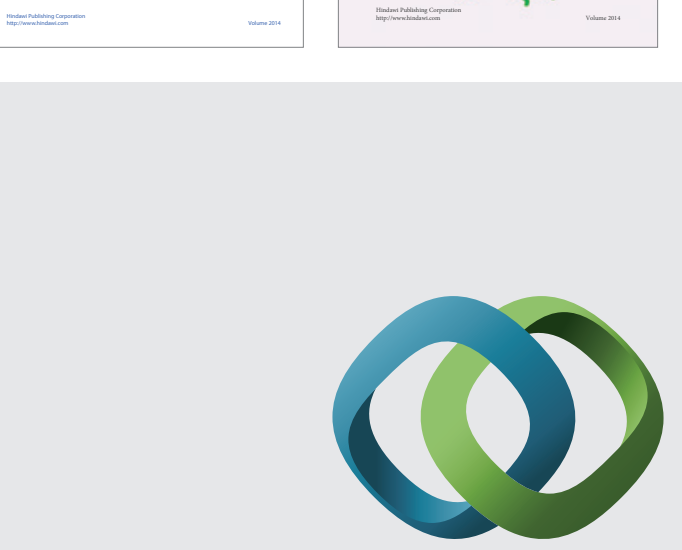

\section{Hindawi}

Submit your manuscripts at

http://www.hindawi.com
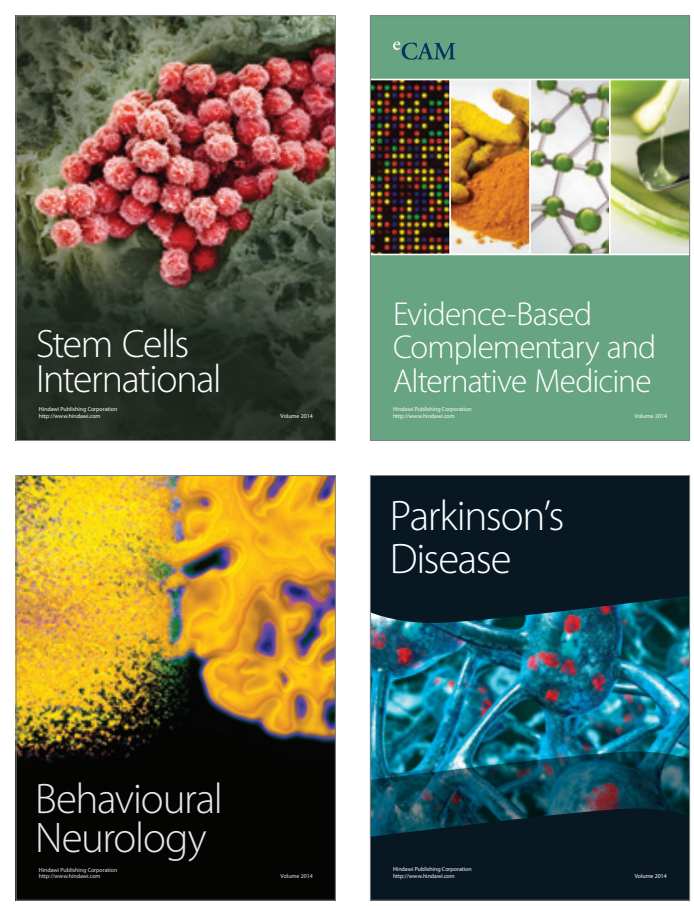

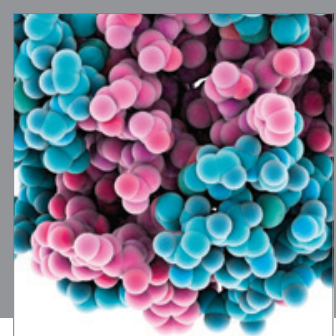

Journal of
Diabetes Research

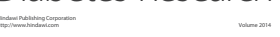

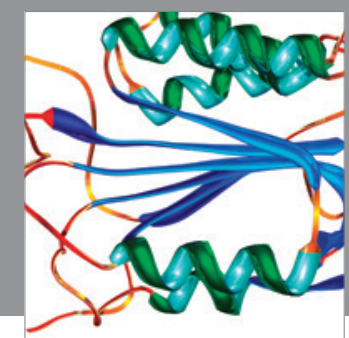

Disease Markers
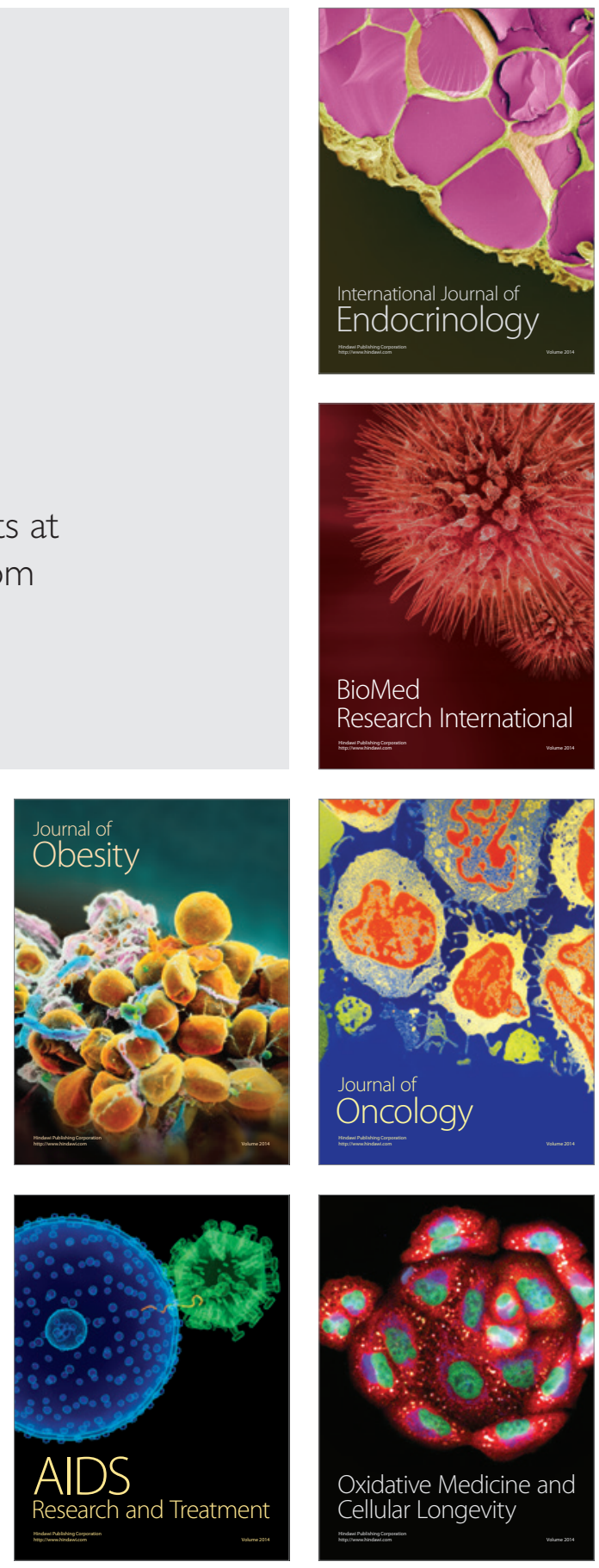